Louisiana State University

LSU Digital Commons

$1-1-2014$

\title{
A comparative study on the risks of radiogenic second cancers and cardiac mortality in a set of pediatric medulloblastoma patients treated with photon or proton craniospinal irradiation
}

\author{
Rui Zhang \\ University of Texas Graduate School of Biomedical Sciences at Houston \\ Rebecca M. Howell \\ University of Texas Graduate School of Biomedical Sciences at Houston \\ Phillip J. Taddei \\ University of Texas Graduate School of Biomedical Sciences at Houston \\ Annelise Giebeler \\ University of Texas Graduate School of Biomedical Sciences at Houston \\ Anita Mahajan \\ University of Texas MD Anderson Cancer Center
}

See next page for additional authors

Follow this and additional works at: https://digitalcommons.Isu.edu/physics_astronomy_pubs

\section{Recommended Citation}

Zhang, R., Howell, R., Taddei, P., Giebeler, A., Mahajan, A., \& Newhauser, W. (2014). A comparative study on the risks of radiogenic second cancers and cardiac mortality in a set of pediatric medulloblastoma patients treated with photon or proton craniospinal irradiation. Radiotherapy and Oncology, 113 (1), 84-88. https://doi.org/10.1016/j.radonc.2014.07.003

This Article is brought to you for free and open access by the Department of Physics \& Astronomy at LSU Digital Commons. It has been accepted for inclusion in Faculty Publications by an authorized administrator of LSU Digital Commons. For more information, please contact ir@lsu.edu. 


\section{Authors}

Rui Zhang, Rebecca M. Howell, Phillip J. Taddei, Annelise Giebeler, Anita Mahajan, and Wayne D. Newhauser 


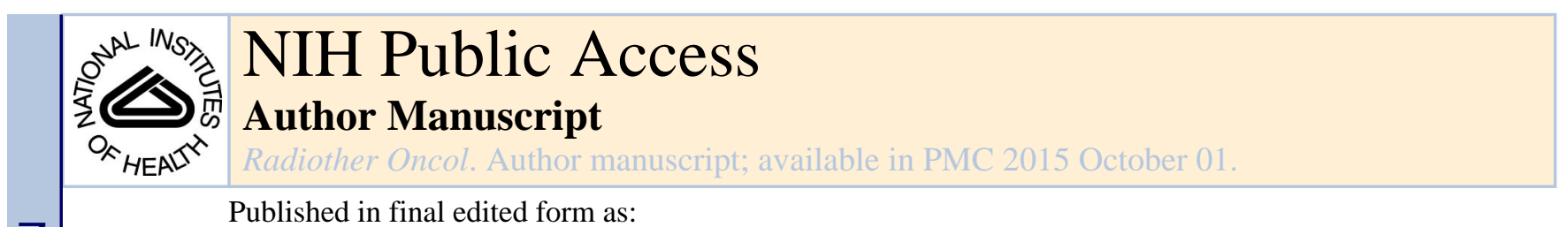

Published in final edited form as:

Radiother Oncol. 2014 October ; 113(1): 84-88. doi:10.1016/j.radonc.2014.07.003.

\title{
A comparative study on the risks of radiogenic second cancers and cardiac mortality in a set of pediatric medulloblastoma patients treated with photon or proton craniospinal irradiation
}

\author{
Rui Zhang ${ }^{a, b,{ }^{,}}$, Rebecca M. Howell ${ }^{a, b}$, Phillip J. Taddej ${ }^{a, b, c}$, Annelise Giebeler ${ }^{a, b}$, Anita \\ Mahajan $^{d}$, and Wayne D. Newhauser ${ }^{a, b, e, \dagger}$ \\ The University of Texas Graduate School of Biomedical Sciences at Houston, Houston, TX, USA \\ bDepartment of Radiation Physics, The University of Texas MD Anderson Cancer Center, \\ Houston, TX, USA \\ 'Department of Radiation Oncology, American University of Beirut Medical Center, Beirut, \\ Lebanon \\ dDepartment of Radiation Oncology, The University of Texas MD Anderson Cancer Center, \\ Houston, TX, USA \\ eMedical Physics Program, Department of Physics and Astronomy, Louisiana State University, \\ Baton Rouge, LA, USA
}

Abstract

Purpose-To compare the risks of radiogenic second cancers and cardiac mortality in 17 pediatric medulloblastoma patients treated with passively scattered proton or field-in-field photon craniospinal irradiation (CSI).

Material/ methods-Standard of care photon or proton CSI treatment plans were created for all 17 patients in a commercial treatment planning system (TPS) (Eclipse version 8.9; Varian Medical Systems, Palo Alto, CA) and prescription dose was $23.4 \mathrm{~Gy}$ or $23.4 \mathrm{~Gy}(\mathrm{RBE})$ to the age specific target volume at $1.8 \mathrm{~Gy} /$ fraction. The therapeutic doses from proton and photon CSI plans were estimated from TPS. Stray radiation doses were determined from Monte Carlo simulations for proton CSI and from measurements and TPS for photon CSI. The Biological Effects of Ionization Radiation VII report and a linear model based on childhood cancer survivor data were used for risk predictions of second cancer and cardiac mortality, respectively.

(C) 2014 Elsevier Ireland Ltd. All rights reserved.

${ }^{\dagger}$ Corresponding author. Department of Physics and Astronomy, Louisiana State University, Baton Rouge, LA, USA Tel.: 225-578-2762; Fax: 225-215-1376 newhauser@1su.edu (W. Newhauser).

*Current address: Mary Bird Perkins Cancer Center, Baton Rouge, LA, USA

Conflict of Interest Statement: The authors have no conflicts of interest.

Publisher's Disclaimer: This is a PDF file of an unedited manuscript that has been accepted for publication. As a service to our customers we are providing this early version of the manuscript. The manuscript will undergo copyediting, typesetting, and review of the resulting proof before it is published in its final citable form. Please note that during the production process errors may be discovered which could affect the content, and all legal disclaimers that apply to the journal pertain. 
Results-The ratios of lifetime attributable risk (RLARs) (proton/photon) ranged from 0.10 to 0.22 for second cancer incidence and ranged from 0.20 to 0.53 for second cancer mortality, respectively. The ratio of relative risk ( $R R R)$ (proton/photon) of cardiac mortality ranged from 0.12 to 0.24 . The RLARs of both cancer incidence and mortality decreased with patient's age at exposure (e), while the $R R R$ s of cardiac mortality increased with $e$. Girls had a significantly higher $R L A R$ of cancer mortality than boys.

Conclusion-Passively scattered proton CSI provides superior predicted outcomes confers lower predicted risks of a second cancer and cardiac mortality than field-in-field photon CSI for all medulloblastoma patients in a large clinically representative sample in the United States, but the magnitude of superiority depend strongly on the patients' anatomical development status.

\section{Keywords}

Medulloblastoma; craniospinal irradiation; proton therapy; second cancer; cardiac mortality

\section{Introduction}

Improvements in pediatric cancer patients treatment outcomes have been clearly demonstrated, including longer mean survival time compared with previous treatment eras $[1,2]$. Radiation therapy has long been recognized as an effective treatment for many cancers in children. However, radiogenic side effects, which include second cancers, cardiac toxicity, pulmonary toxicity, and impaired growth and bone development [3,4], may reduce survivors' lifespan and quality of life.

Second cancers account for around $16 \%$ of all cancers and solid tumors comprise a leading cause of mortality among cancer survivors in the United States [5]. For some pediatric cancers, treatment-induced second cancers can cause more deaths than the primary cancers do [6]. The Childhood Cancer Survivor Study (CCSS), which included a large cohort of cancer patients who have survived more than five years, reported that the mortality rate attributable to recurrence or progression of primary cancers in children is decreasing while the mortality rates attributable to subsequent neoplasms and to cardiac and pulmonary toxicity from treatment are increasing [7]. CCSS also found that the risk of cardiovascular disease was substantially higher in cancer survivors than in the general population [7-9] and that cardiovascular events are the leading non-malignant cause of death among survivors of childhood cancer [10].

The risks of radiogenic second cancer or cardiac toxicity have been studied [11-15], but few of those studies compared photon and proton radiation therapies in a large sample set of patients. The aim of this work was to predict and compare the risks of radiogenic second cancers and cardiac mortality in a representative sample of pediatric patients with medulloblastoma (MB) treated using photon craniospinal irradiation (CSI) versus proton CSI. Both therapeutic and secondary radiation doses were included in the risk calculations. 


\section{Material and methods}

\section{Patient selection}

Pediatric patients diagnosed with MB and treated with proton radiation therapy at The University of Texas MD Anderson Cancer Center (UT MDACC) in 2007-2009 were selected for this study We followed a retrospective data analysis protocol approved by our institutional review board. Inclusion criteria were an age at exposure of 2-18 years, treatment in the supine position and available computed tomography (CT) images. Seventeen MB patients were included: 10 who were 2 to 10 years old and 7 who were 11 to 18 years old. Among them 8 were females and 9 were males. The patients were selected to represent a general population of children and adolescents who received radiation therapy for MB; our sample included males and females whose ages, heights, and weights spanned a clinically relevant and representative spectrum (age 2 to 18 years, height 85 to $173 \mathrm{~cm}$, body mass index (BMI) 16.4 to $37.9 \mathrm{~kg} / \mathrm{m}^{2}$ ).

\section{Treatment techniques and organs of interest}

Proton and photon treatment plans were both retrospectively created using a commercial TPS (Eclipse version 8.9; Varian Medical Systems, Palo Alto, CA). The proton treatment plans were designed to treat the patient using the passively scattered proton beam line at UT MDACC proton therapy center. For both plans, an age-specific target volume was defined for each patient. This volume included the brain, the spinal canal, and the entire vertebral body for patients younger than 15 years (to prevent bone growth deformity due to nonuniform dose distribution in the vertebral body), and only the brain and spinal canal for patients 15 years old or older (with a 2-3 mm margin anteriorly). Typical target volumes for representative patients were shown in previous reports [16,17]. The proton treatment plans included right and left posterior oblique cranial fields and 1-3 posterior-anterior spinal fields, depending on the length of the patient's spinal axis. The photon treatment plans contained 2 opposed lateral cranial fields and 1 or 2 posterior-anterior spinal fields, depending on the length of the spinal axis. All photon fields were $6 \mathrm{MV}$, and a field-in-field technique was used to improve the dose uniformity across the target volume. The treatment plans were designed to deliver 23.4 Gy (relative biological effectiveness [RBE]) (proton beam RBE of 1.1 was used here [18]) for proton CSI or 23.4 Gy for photon CSI to the age specific target volume at $1.8 \mathrm{~Gy} /$ fraction. Additional details about the proton and photon radiation therapy plans and typical isodose distributions for representative patients can be found in previous reports [16,17].

The organs of interest for radiogenic second cancer risk estimation included the stomach, colon, lungs, breasts, bladder, thyroid, liver, prostate, and remainder (i.e., the organs and tissues for which risk coefficients were not provided in the Biological Effects of Ionizing Radiation [BEIR] VII report [19]). These organs as well as each patient's heart were contoured for this study in their entirety within the extent of the CT images. The ovaries were not included in our risk analysis because their position is highly variable and they are not readily visible on CT images [20]. The external surface of the heart was contoured in every CT slice from the inferior border of the right pulmonary artery to the apex of the heart. 


\section{Dose reconstruction and risk calculation}

The therapeutic doses from the proton and photon treatment plans were estimated from TPS directly. The stray dose from proton therapy was calculated by our Monte Carlo Proton Radiotherapy Treatment Planning system [11], which uses the Monte Carlo N-particle eXtended code (version 2.6; Los Alamos National Laboratory, Los Alamos, NM) [21] as a dose calculation engine. The secondary dose from photon therapy was obtained from the TPS and measurement [22]. Our method was previously described in the literature [23,24] and briefly summarized here: For organs in close proximity to the treatment field, doses are accurately reported by the TPS and as such doses were directly taken from the TPS. For organs far from the treatment field, TPS data were supplemented using analytical model based on measurements in an anthropomorphic phantom. The equivalent dose in each organ, $H_{T}$, was calculated by multiplying mean organ dose, $D_{T}$, by the radiation weight factor, $\overline{w_{R}}$. For stray neutrons in proton CSI, $\overline{w_{R}}$ values were taken from a separate CSI study by Newhauser et al. [11].

Lifetime attributable risk ( $L A R)$ was defined in BEIR VII as the probability of cancer incidence or mortality during one's lifetime (living to 100 years) after exposure to a certain equivalent dose $\left(H_{T}\right)$ at age at exposure, $e$. For each modality, the total $L A R$ was calculated as follows:

$$
L A R_{\text {modality }}=\left(\sum_{T} L A R_{T}\right)_{\text {modality }}
$$

where the sum is over all the organs of interest. To compare the risks of proton and photon therapies, the ratio of LARs (RLAR) between the two modalities was defined as follows:

$$
R L A R=L A R_{h} / L A R_{p} \quad \text { (2) }
$$

where the subscripts $h$ and $p$ denote proton therapy and photon therapy, respectively More details of the risk calculation for second cancers can be found in Zhang et al. [14].

Tukenova et al. [25] recently reported a linear relationship between the mean radiation dose to the heart and the relative risk of cardiac mortality based on follow-up data from a large sample of childhood cancer survivors:

$$
R R=1+\alpha_{1} H \quad(3)
$$

where $R R$ is the relative risk; $H$ is the mean heart equivalent dose; and $a_{1}$, the linear coefficient, is 0.6 (95\% confidence interval, 0.2-2.5). The ratio of relative risk (RRR) of cardiac mortality was defined as

$$
R R R=R R_{h} / R R_{p}
$$

the subscript $h, p$ denotes proton therapy and photon therapy, respectively. 


\section{Statistical analysis}

A two-sided linear correlation was used to test for significant correlations between calculated values of $R L A R, R R, R R R$ and patients' age at exposure. The two-sample twosided $t$-test was used to test differences between sexes. A $p$ value of less than 0.05 indicates a significant correlation or a significant difference in these tests. All statistical analyses were performed with MATLAB (Mathworks, Natick, MA).

\section{Results}

Table 1 lists the mean primary organ doses in current study and those from Mu et al. [12]. Except in lung, proton plans resulted in much lower organ doses than photon plans. The stray radiation dose is also an important component of a full comparison of different radiotherapy modalities, especially when late effects like second cancer and cardiac toxicity are considered. Table 1 also lists the mean stray neutron doses by organ from the proton plans. Owing to the methodology we used to calculate photon dose, it was not possible to separate the stray (secondary) dose from the therapeutic dose for photon plans. Specifically, the treatment planning algorithm does not distinguish between primary, scattered, and leakage photons.

Table 2 and 3 (supplemental material) show the organ-specific $L A R$ values of cancer incidence and mortality (i.e., a fatal radiation-induced cancer) after proton or photon CSI for the sample of patients. The predominant cancer risks were from lung and remainder for both cancer incidence and mortality. Photon CSI confers much higher risks to all the organs for all the patients.

Fig. 1 shows the RLAR of second cancer incidence and mortality for the sample of patients as a function of patient age at exposure (e). The RLARs were less than 1 for each patient, regardless of $e$ and sex, which means proton therapy conferred significantly lower risks of cancer incidence and mortality than photon therapy ( $p \ll 0.001$ for both). The RLARs ranged from 0.10 to 0.22 for second cancer incidence and ranged from 0.20 to 0.53 for second cancer mortality, respectively. There was a significant correlation between predicated $R L A R$ of cancer incidence and $e$ (correlation coefficient $r=-0.74 ; p<0.001$ ), and RLAR of second cancer mortality and $e$ were weakly correlated $(r=-0.44 ; p=0.075)$. The predicted $R L A R$ of second cancer incidence was independent of sex ( $t$ test, $p=0.29$ ), but interestingly, the predicted $R L A R$ of second cancer mortality was dependent on sex: girls had a significantly higher $R L A R$ of second cancer mortality than boys ( $t$ test, $p=0.023$ ).

Fig. 2 shows the $R R$ and $R R R$ of cardiac mortality for this sample of patients as a function of $e$. The $R R R$ s of cardiac mortality ranged from 0.12 to 0.24 . Proton therapy conferred significantly lower risks of cardiac mortality than photon therapy $(p<0.001)$. There was no significant correlation between predicated $R R$ values and $e$ for proton CSI $(r=-0.18, p=$ $0.48)$, but there was a significant correlation between $R R$ and $e$ for photon CSI $(r=-0.70, p$ $=0.0019)$, and there was a significant correlation between $R R R$ and $e(r=0.60, p=0.01)$. The predicted $R R$ values were independent of sex ( $t$ test, $p=0.37$ for proton CSI and $p=$ 0.76 for photon CSI), and $R R R$ values were independent of $\operatorname{sex}(t$ test, $p=0.55)$. 


\section{Discussion}

We predicted risks of radiogenic second cancers and cardiac mortality after photon or proton CSI for a sample of 17 pediatric patients with $\mathrm{MB}$, considering both primary and stray radiation doses. We found that proton CSI can reduce the predicted risks of radiogenic second cancer incidence and cardiac mortality for these pediatric patients by 6 times, and can reduce the predicted risk of second cancer mortality by 3 times, compared with conventional photon CSI.

Considering that there are around 600 new MB cases each year in the United States [26], that CSI is a mainstay of therapy [27], and a clinically representative sample used in this work, our findings are of clinical significance because they show that proton CSI can potentially confer much lower risks of late effects than photon CSI for all these MB patients in the United States.

$R L A R$ s of second cancer incidence and mortality decreased with increasing $e$, because the target volume included the entire vertebral body for the younger patients but only the brain and spinal canal for the older patients, and because normal tissues are further away from treatment site in bigger patients. Although the proton and photon CSI plans both followed these mechanisms, the inherent physical characteristic of proton beams (stop sharply after target volume) results in a lower exit dose to normal tissues in the older patients than the photon beams did. These indicated that older patients could benefit more from proton CSI in terms of reducing predicted second cancer.

Girls had significantly higher RLARs of second cancer mortality than boys, partially because the lung dose from proton CSI was significantly less than that from photon CSI for boys ( $p$ $=0.015)$ but not for girls $(p=0.07)$. In fact, girls generally have smaller size than boys at the same age, which means a larger portion of girl's lung was exposed to therapeutic radiation and thus lung dose difference between proton and photon CSI was not that obvious. Together with the facts that lung dose was higher than other organs (Table 1) and it has the highest risk coefficient for second cancer mortality of any organ considered in this work [19], the lung dose contributed to the girls' higher RLARs of second cancer mortality.

The $R R$ of cardiac mortality decreased with $e$ after photon CSI but not after proton CSI. This is because the heart dose was not affected substantially by patient size in proton CSI as in photon CSI (in other words, there was almost no exit proton beam dose to the heart, even for the young patients), so the risk after proton CSI did not decrease significantly with $e$ (fig. 2(a)). This also explained why $R R R$ values increased with $e$, and indicated younger patints could benefit more from proton CSI in terms of reducing predicted cardiac mortality.

This study had a number of strengths. We included a larger cohort of MB patients than previous studies did, with ages and sexes that are representative of a general pediatric population in the United States. Because each treatment technique was based on the current standard of care at a single institution, our results were clinically realistic. In addition, our advanced dose reconstruction tools, including TPS, Monte Carlo simulation and phantom measurements, enabled us to provide an accurate and comprehensive evaluation of radiation doses. 
We can compare our dosimetric data with those of Mu et al. [12], who used conventional photon therapy plans and intensity modulated proton therapy (IMPT) plans in a study of 5 pediatric MB patients. Because Mu et al. did not include stray radiation doses in their study, we can compare only the therapeutic doses (Table 1). The two sets of dosimetric results agree well, and both studies found that proton beams delivered a lower primary dose to all the organs at risk than photon beams. Because Mu et al. used effective dose to estimate second cancer risk, we cannot compare their risk estimates with ours directly.

Brodin et al. [13] compared various treatment techniques, including IMPT and 3D conformal photon therapy, for 10 pediatric MB patients. Because they did not publish the organ dose values and used different dose-risk models for second cancer and cardiac mortality it is difficult to compare our results with theirs directly. However, we can compare the ratios of lifetime risk of second cancer incidence: their mean ratio of risk between IMPT and $3 \mathrm{D}$ conformal photon therapy was 0.16 , which agrees well with our cohort's mean RLAR of second cancer incidence after proton versus photon therapy $(0.15 \pm 0.04)$.

Our study had some potential limitations. First, no consensus exists on which second cancer dose-risk models should be used for organs that receive high doses. Some groups have argued that the BEIR VII report was developed for low dose and low dose rate and proposed alternative dose-risk models [28-31]. However, these new risk models are still under development and may contain unknown large errors. We therefore decided to follow the BEIR VII report and use ratios of risks instead of absolute risk values of second cancer because several independent studies suggested the former to be a more robust measure for comparing treatment modalities [13,14,32,33]. The good agreement of $R L A R$ values between Brodin et al. [13] and the present work provided more strong evidence that a ratio of risks should be used for inter-institution comparisons. The second limitation is that we only investigated a passively scattered proton CSI and field-in-field photon CSI in this work, while other modalities like IMPT [13,34], IMRT [34,35], Tomotherapy [35,36] and Volumetric Modulated Arc Therapy (VMAT) [37] are also used at some centers. However, most of these advanced treatment modalities are not routinely used for CSI treatment, whereas the techniques in our study are. Also, with respect to the photon treatment planning, we used advanced conformal therapy, i.e., the field-in-field modulation technique to minimize heterogeneities in the dose distributions. This had the effect of reducing the plan hot spots and for the spine fields resulted in a marked decrease in the dose to organs anterior to the spinal canal. So we don't consider this as a real limitation but strength because our results are based on clinically realistic data and treatment plans. Another potential limitation is that we estimated the $R R$ of cardiac mortality only for the entire heart instead of calculating risks for each heart sub-structure, e.g., myocardium and pericardium [38]. This was because the literature did not specifically report any detailed relationship between dose and outcome of cardiac mortality for individual heart sub-structures in MB patients, so normal tissue complication probability (NTCP) model parameters were not available. Considering the possible large uncertainties associated with NTCP calculations, we decided for the purpose of this study that $R R$ based on whole-heart dose and a linear risk model was a more suitable metric. 


\section{Conclusion}

We performed dose reconstructions and predictions of the risks of radiogenic second cancer and cardiac mortality for a clinically representative sample of MB patients. The results revealed that proton CSI can confer much lower predicted risks of late effects than photon CSI. These findings strongly suggest proton therapy will provide better quality of life for long-term survivors of pediatric MB..

\section{Supplementary Material}

Refer to Web version on PubMed Central for supplementary material.

\section{Acknowledgments}

The authors are grateful to Melissa Burkett and Sarah Bronson for their assistance in editing this manuscript. This work was supported by a Sowell-Huggins Scholarship (RZ), a President Research Scholarship (RZ), the National Cancer Institute (award 1R01CA131463-01A1) (WDN) and a subcontract of that award (RMH), Northern Illinois University through a subcontract of a Department of Defense contract (award W81XWH-08-1-0205) (WDN), and the Fogarty International Center at the National Institutes of Health (award K01TW008409) (PJT).

\section{References}

1. Jemal A, Siegel R, Ward E, et al. Cancer statistics, 2008. CA Cancer J Clin. 2008; 58:71-96. [PubMed: 18287387]

2. Hewitt, M.; Weiner, SL.; Simone, JVE. Childhood Cancer Survivorship: Improving care and quality of life. Washington, D.C: National Academies Press; 2003.

3. Fossati P, Ricardi U, Orecchia R. Pediatric medulloblastoma: toxicity of current treatment and potential role of protontherapy. Cancer Treat Rev. 2009; 35:79-96. [PubMed: 18976866]

4. NCRP, Report No 170: Second primary cancers and cardiovascular disease after radiation therapy. 2011

5. Wood ME, Vogel V, Ng A, Foxhall L, Goodwin P, Travis LB. Second malignant neoplasms: assessment and strategies for risk reduction. J Clin Oncol. 2012; 30:3734-3745. [PubMed: 23008293]

6. Tubiana M. Can we reduce the incidence of second primary malignancies occurring after radiotherapy? A critical review Radiother Oncol. 2009; 91:4-15. discussion 11-13.

7. Armstrong GT, Liu Q, Yasui Y, et al. Late mortality among 5-year survivors of childhood cancer: a summary from the Childhood Cancer Survivor Study. J Clin Oncol. 2009; 27:2328-2338. [PubMed: 19332714]

8. Oeffinger KC, Mertens AC, Sklar CA, et al. Chronic health conditions in adult survivors of childhood cancer. N Engl J Med. 2006; 355:1572-1582. [PubMed: 17035650]

9. Lipshultz SE, Adams MJ. Cardiotoxicity after childhood cancer: beginning with the end in mind. J Clin Oncol. 2010; 28:1276-1281. [PubMed: 20142585]

10. Mulrooney DA, Yeazel MW, Kawashima T, et al. Cardiac outcomes in a cohort of adult survivors of childhood and adolescent cancer: retrospective analysis of the Childhood Cancer Survivor Study cohort. BMJ. 2009; 339:b4606. [PubMed: 19996459]

11. Newhauser WD, Fontenot JD, Mahajan A, et al. The risk of developing a second cancer after receiving craniospinal proton irradiation. Phys Med Biol. 2009; 54:2277-2291. [PubMed: 19305036]

12. Mu X, Bjoerk-Eriksson T, Nill S, et al. Does electron and proton therapy reduce the risk of radiation induced cancer after spinal irradiation for childhood medulloblastoma? A comparative treatment planning study Acta Oncologica. 2005; 44:554-562. 
13. Brodin NP, Rosenschold PM, Aznar MC, et al. Radiobiological risk estimates of adverse events and secondary cancer for proton and photon radiation therapy of pediatric medulloblastoma. Acta Oncol. 2011; 50:806-816. [PubMed: 21767178]

14. Zhang R, Howell RM, Giebeler A, Taddei PJ, Mahajan A, Newhauser WD. Comparison of risk of radiogenic second cancer following photon and proton craniospinal irradiation for a pediatric medulloblastoma patient. Phys Med Biol. 2013; 58:807-823. [PubMed: 23322160]

15. Taddei PJ, Mahajan A, Mirkovic D, et al. Predicted risks of second malignant neoplasm incidence and mortality due to secondary neutrons in a girl and boy receiving proton craniospinal irradiation. Phys Med Biol. 2010; 55:7067-7080. [PubMed: 21076189]

16. Howell RM, Giebeler A, Koontz-Raisig W, et al. Comparison of therapeutic dosimetric data from passively scattered proton and photon craniospinal irradiations for medulloblastoma. Radiat Oncol. 2012; 7:116-127. [PubMed: 22828073]

17. Giebeler A, Newhauser WD, Amos RA, Mahajan A, Homann K, Howell RM. Standardized treatment planning methodology for passively scattered proton craniospinal irradiation. Radiat Oncol. 2013; 8:32. [PubMed: 23375151]

18. ICRU, Prescribing, Recording, and Reporting Proton-Beam Therapy ICRU Report 78. 2007

19. NRC, Health Risks from Exposure to Low Levels of Ionizing Radation: BEIR VII - Phase 2. Nation Research Council of the National Academies. 2006

20. Perez-Andujar A, Newhauser WD, Taddei PJ, Mahajan A, Howell RM. The predicted relative risk of premature ovarian failure for three radiotherapy modalities in a girl receiving craniospinal irradiation. Phys Med Biol. 2013; 58:3107-3123. [PubMed: 23603657]

21. Hendricks JS, McKinney GW, Durkee JW, et al. MCNPX, Version 26c. 2006

22. Taddei PJ, Jalbout W, Howell RM, et al. Analytical model for out-of-field dose in photon craniospinal irradiation. Phys Med Biol. 2013; 58:7463-7479. [PubMed: 24099782]

23. Howell RM, Scarboro SB, Taddei PJ, Krishnan S, Kry SF, Newhauser WD. Methodology for determining doses to in-field, out-of-field and partially in-field organs for late effects studies in photon radiotherapy. Phys Med Biol. 2010; 55:7009-7023. [PubMed: 21076193]

24. Howell RM, Scarboro SB, Kry SF, Yaldo DZ. Accuracy of out-of-field dose calculations by a commercial treatment planning system. Phys Med Biol. 2010; 55:6999-7008. [PubMed: 21076191]

25. Tukenova M, Guibout C, Oberlin O, et al. Role of cancer treatment in long-term overall and cardiovascular mortality after childhood cancer. J Clin Oncol. 2010; 28:1308-1315. [PubMed: 20142603]

26. CBTRUS, Statistical report: Primary brain tumors in the United States, 2004-2008. 2012

27. Packer RJ, Vezina G. Management of and prognosis with medulloblastoma: therapy at a crossroads. Arch Neurol. 2008; 65:1419-1424. [PubMed: 19001159]

28. Shuryak I, Hahnfeldt P, Hlatky L, Sachs RK, Brenner DJ. A new view of radiation-induced cancer: integrating short- and long-term processes. Part I: approach. Radiation and environmental biophysics. 2009; 48:263-274. [PubMed: 19536557]

29. Shuryak I, Hahnfeldt P, Hlatky L, Sachs RK, Brenner DJ. A new view of radiation-induced cancer: integrating short- and long-term processes. Part II: second cancer risk estimation. Radiation and environmental biophysics. 2009; 48:275-286. [PubMed: 19499238]

30. Schneider U, Sumila M, Robotka J. Site-specific dose-response relationships for cancer induction from the combined Japanese A-bomb and Hodgkin cohorts for doses relevant to radiotherapy. Theoretical biology \& medical modelling. 2011; 8:27. [PubMed: 21791103]

31. Schneider U, Sumila M, Robotka J, Gruber G, Mack A, Besserer J. Dose-response relationship for breast cancer induction at radiotherapy dose. Radiat Oncol. 2011; 6:67. [PubMed: 21651799]

32. Paganetti H, Athar BS, Moteabbed M, AA J, Schneider U, Yock TI. Assessment of radiationinduced second cancer risks in proton therapy and IMRT for organs inside the primary radiation field. Phys Med Biol. 2012; 57:6047-6061. [PubMed: 22968191]

33. Hillbrand M, Georg D, Gadner H, Potter R, Dieckmann K. Abdominal cancer during early childhood: a dosimetric comparison of proton beams to standard and advanced photon radiotherapy. Radiother Oncol. 2008; 89:141-149. [PubMed: 18692928] 
34. Mu X, Bjork-Eriksson T, Nill S. Does electron and proton therapy reduce the risk of radiation induced cancer after spinal irradiation for childhood medulloblastoma? A comparative treatment planning study. Acta Oncol. 2005; 44:554-562. [PubMed: 16165914]

35. Sharma DS, Gupta T, Jalali R, Master Z, Phurailatpam RD, Sarin R. High-precision radiotherapy for craniospinal irradiation: evaluation of three-dimensional conformal radiotherapy, intensitymodulated radiation therapy and helical TomoTherapy. Br J Radiol. 2009; 82:1000-1009. [PubMed: 19581313]

36. Mesbah L, Matute R, Usychkin S, et al. Helical tomotherapy in the treatment of pediatric malignancies: a preliminary report of feasibility and acute toxicity. Radiat Oncol. 2011; 6:102. [PubMed: 21871078]

37. Fogliata A, Bergstrom S, Cafaro I, et al. Cranio-spinal irradiation with volumetric modulated arc therapy: a multi-institutional treatment experience. Radiother Oncol. 2011; 99:79-85. [PubMed: 21421273]

38. Zhang R, Howell RM, Homann K, et al. Predicted risks of radiogenic cardiac toxicity in two pediatric patients undergoing photon or proton radiotherapy. Radiat Oncol. 2013; 8:184. [PubMed: 23880421] 


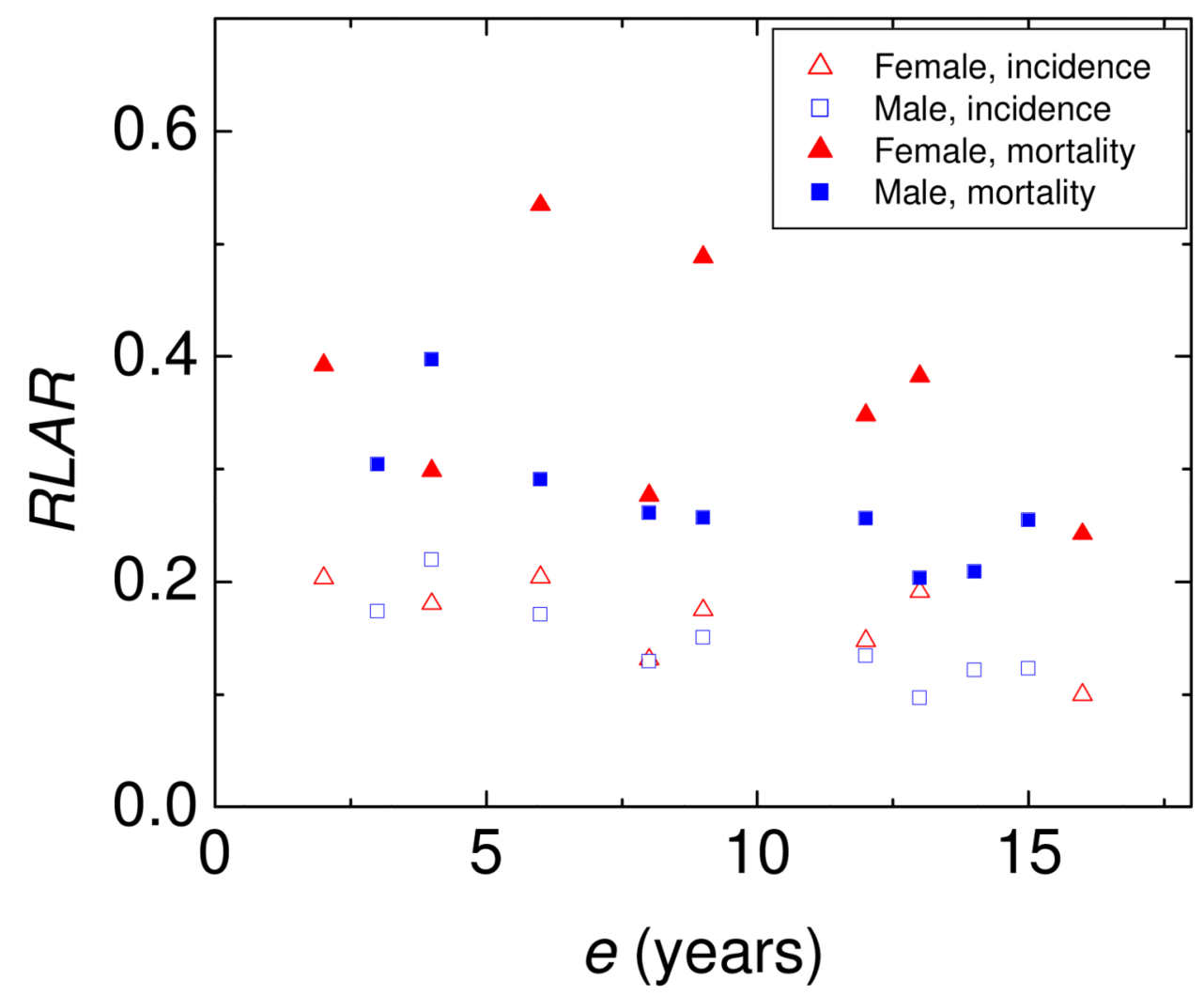

Figure 1.

Ratio of lifetime absolute risk (RLAR) of radiogenic second cancer incidence or mortality after proton versus photon CSI as a function of patient age at exposure, $e$, for a sample of pediatric MB patients. 


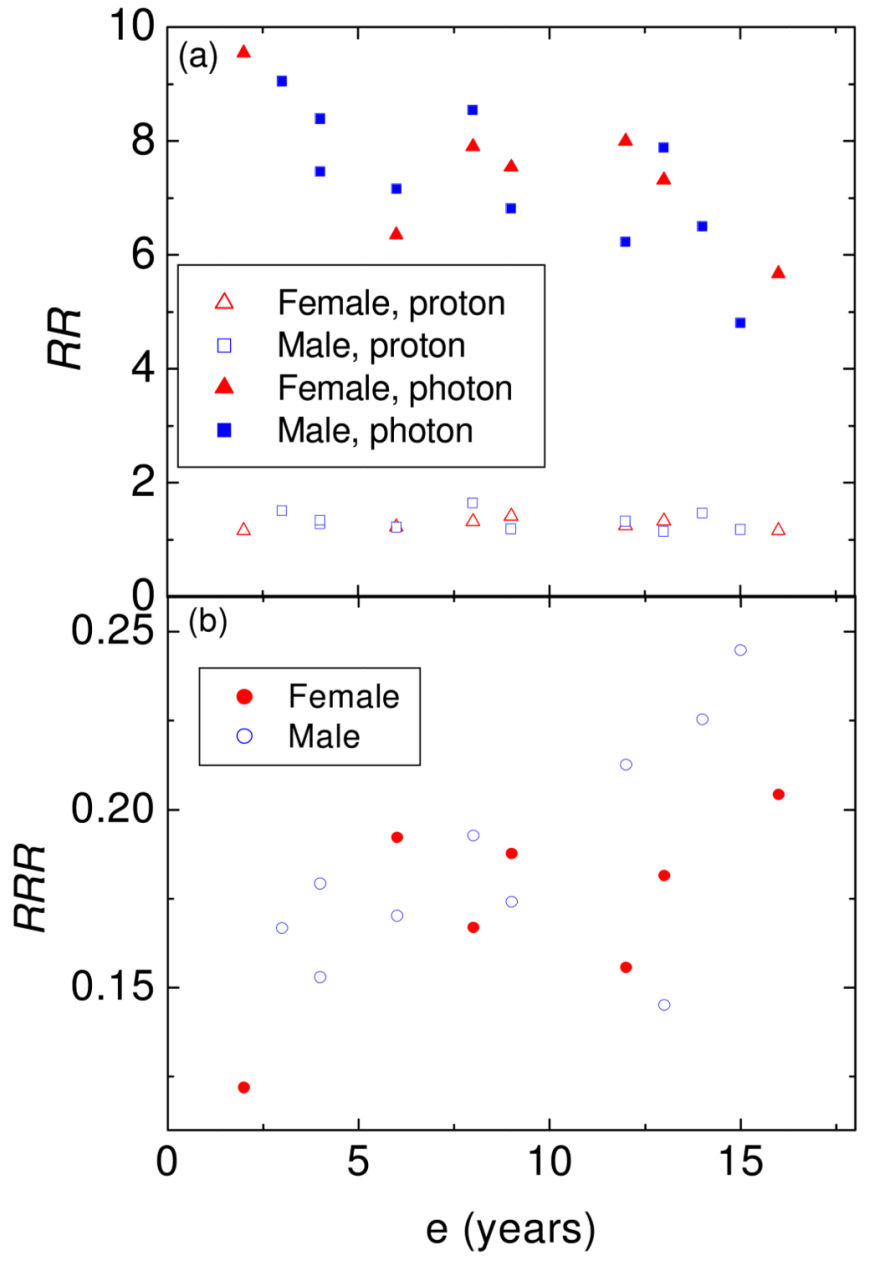

Figure 2.

(a) Relative risk $(R R)$ and (b) ratio of relative risk $(R R R)$ of cardiac mortality after proton or photon CSI as a function of patient age at exposure, $e$, for a sample of pediatric MB patients. 


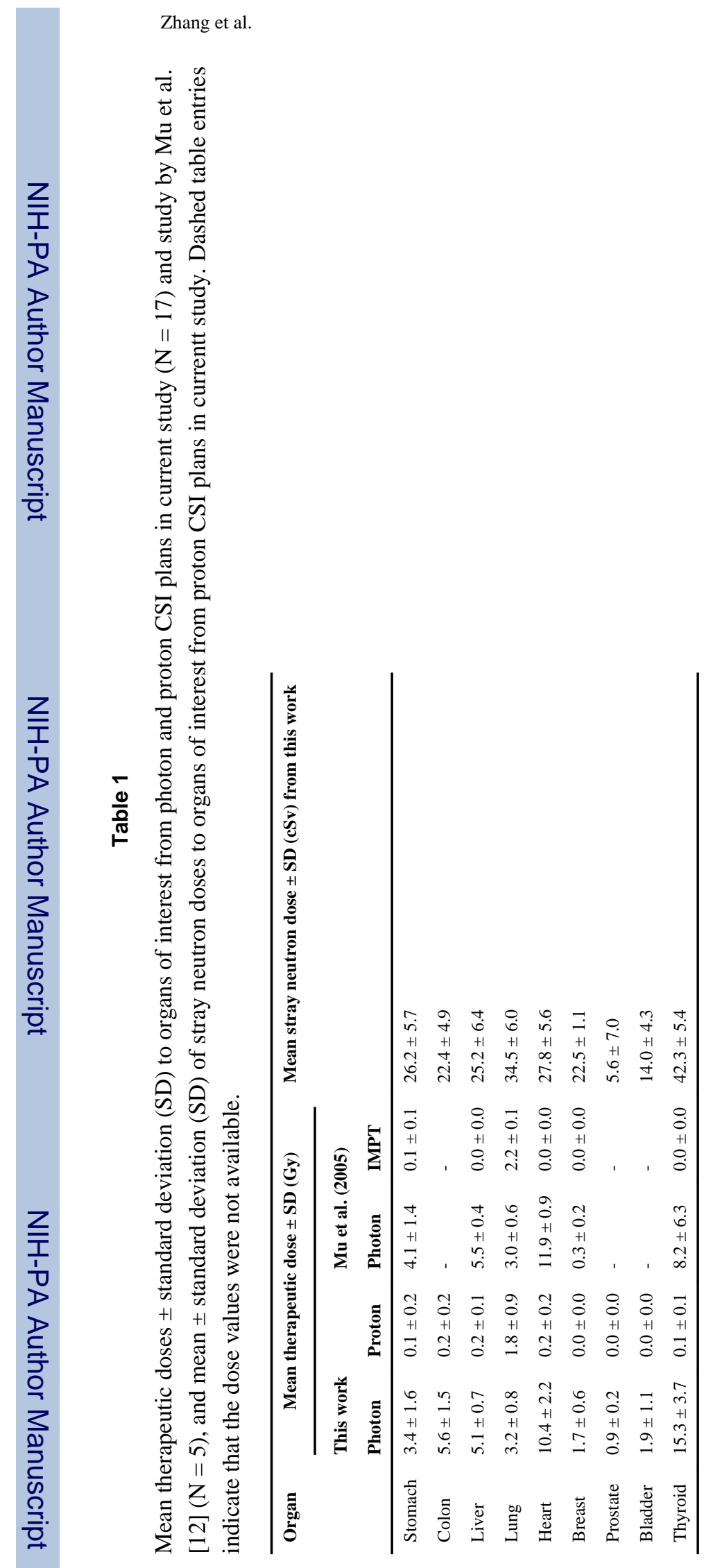

Page 13

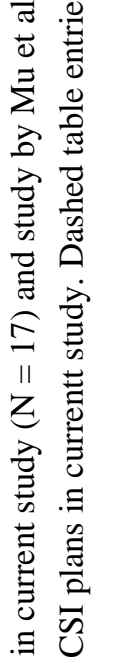

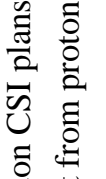

苛

ส

흄ำ

党

幽

范

荡 离

$\stackrel{\circ}{\circ}$

क्षे क्षे

월

वे 웅

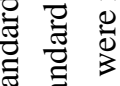

$+1+1$ 孪

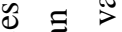

8

: 롱

長的

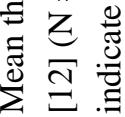

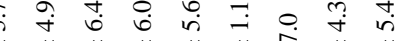

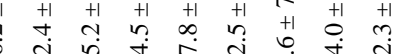

त ते ले ते ते का 


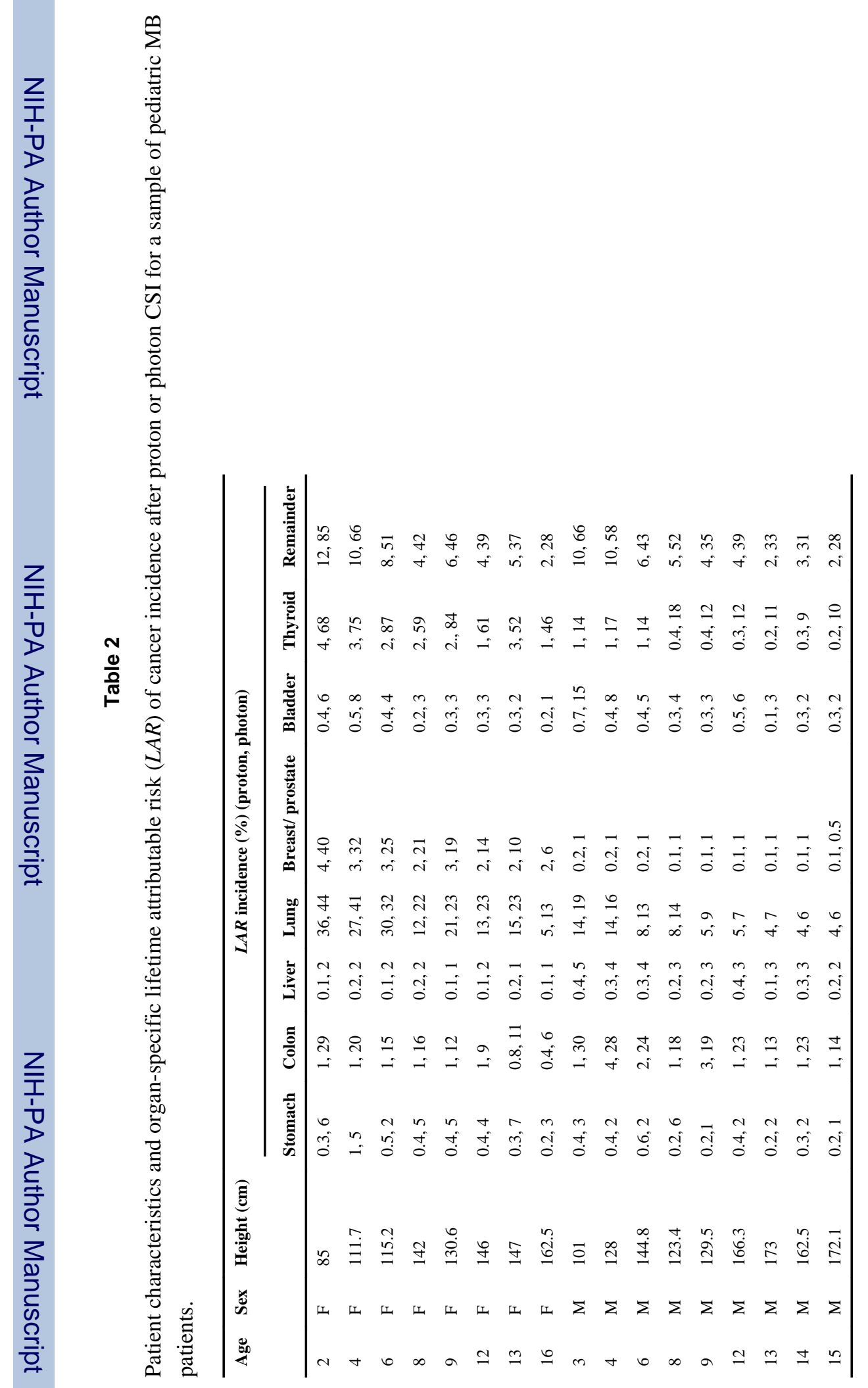

Radiother Oncol. Author manuscript; available in PMC 2015 October 01. 


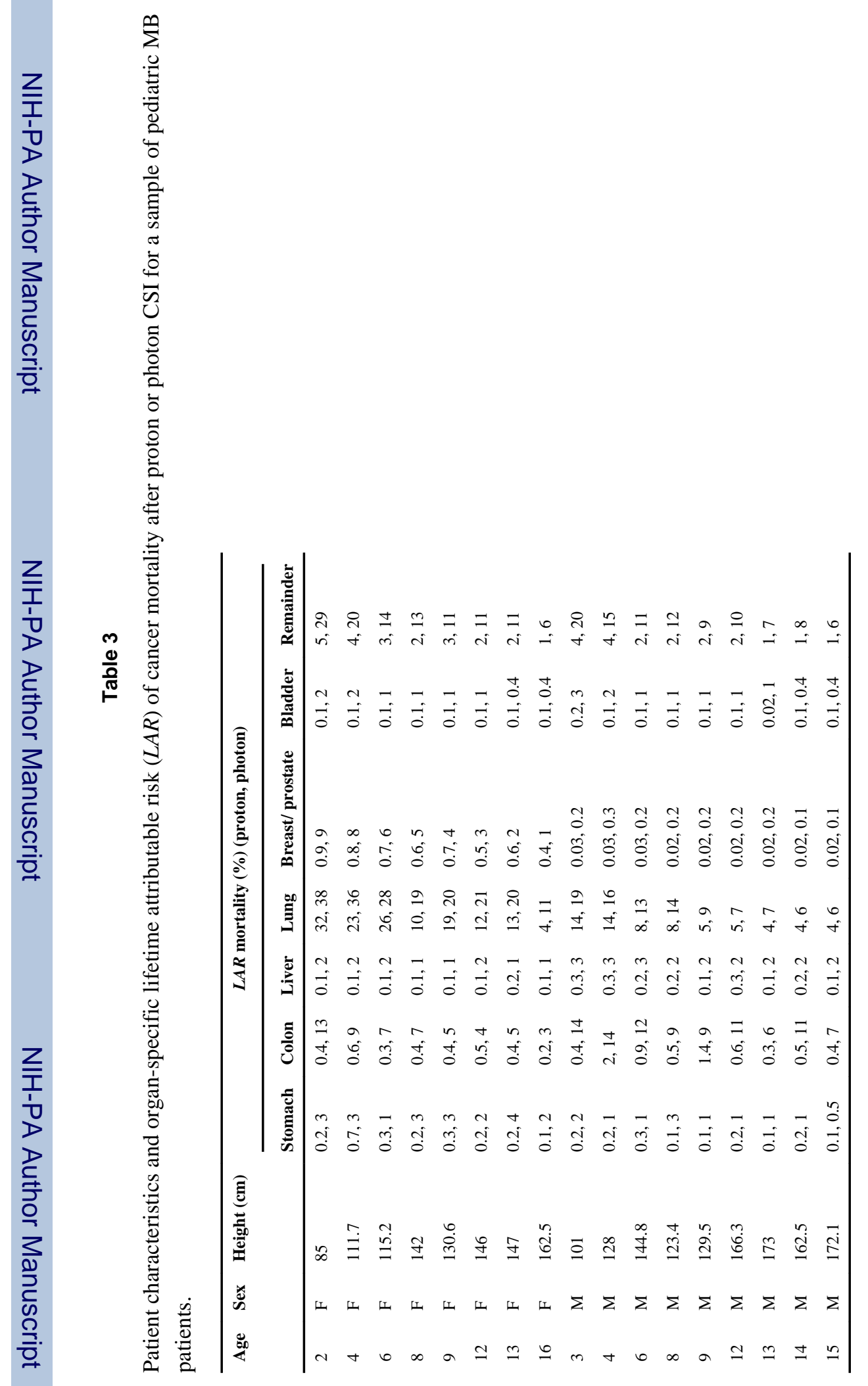

